ARTICLE

\title{
Out-of-equilibrium processes in crystallization of organic-inorganic perovskites during spin coating
}

\author{
Shambhavi Pratap (1) 1,2, Finn Babbe (1) 3, Nicola S. Barchi 4,5, Zhenghao Yuan (1) 4,6, Tina Luong4 ${ }^{4}$, Zach Haber ${ }^{4}$, \\ Tze-Bin Song (1D) ${ }^{4}$, Jonathan L. Slack ${ }^{2}$, Camelia V. Stan (1) ${ }^{2,7}$, Nobumichi Tamura (1) ${ }^{2}$, \\ Carolin M. Sutter-Fella (i) ${ }^{4,8}{ }^{凶}$ \& Peter Müller-Buschbaum (id ${ }^{1,9 凶}$
}

Complex phenomena are prevalent during the formation of materials, which affect their processing-structure-function relationships. Thin films of methylammonium lead iodide $\left(\mathrm{CH}_{3} \mathrm{NH}_{3} \mathrm{Pbl}{ }_{3}, \mathrm{MAPI}\right)$ are processed by spin coating, antisolvent drop, and annealing of colloidal precursors. The structure and properties of transient and stable phases formed during the process are reported, and the mechanistic insights of the underlying transitions are revealed by combining in situ data from grazing-incidence wide-angle $\mathrm{X}$-ray scattering and photoluminescence spectroscopy. Here, we report the detailed insights on the embryonic stages of organic-inorganic perovskite formation. The physicochemical evolution during the conversion proceeds in four steps: i) An instant nucleation of polydisperse MAPI nanocrystals on antisolvent drop, ii) the instantaneous partial conversion of metastable nanocrystals into orthorhombic solvent-complex by cluster coalescence, iii) the thermal decomposition (dissolution) of the stable solvent-complex into plumboiodide fragments upon evaporation of solvent from the complex and iv) the formation (recrystallization) of cubic MAPI crystals in thin film.

\footnotetext{
${ }^{1}$ Physik-Department, Lehrstuhl für Funktionelle Materialien, Technische Universität München, Garching, Germany. ${ }^{2}$ Advanced Light Source, Lawrence Berkeley National Laboratory, Berkeley, USA. ${ }^{3}$ Chemical Sciences Division, Joint Center for Artificial Photosynthesis, Lawrence Berkeley National Laboratory, Berkeley, USA. ${ }^{4}$ Chemical Sciences Division, Lawrence Berkeley National Laboratory, Berkeley, USA. ${ }^{5}$ Laboratoire des Matériaux Semiconducteurs, École Polytechnique Fédérale de Lausanne, Lausanne, Switzerland. ${ }^{6}$ Department of Chemistry, The Pennsylvania State University, University Park, PA, USA. ${ }^{7}$ NIF \& Photon Science, Lawrence Livermore National Laboratory, Livermore, USA. ${ }^{8}$ Molecular Foundry, Lawrence Berkeley National Laboratory, Berkeley, USA.

${ }^{9}$ Heinz Maier-Leibnitz-Zentrum, Technische Universität München, Garching, Germany. ${ }^{凶}$ email: csutterfella@lbl.gov; muellerb@ph.tum.de
} 
$\mathrm{M}$ etal halide-based hybrid perovskite materials have attracted significant research and development interest due to their truly impressive and broad applicability as functional materials ${ }^{1}$. The techno-economic advantages ${ }^{2}$ of hybrid perovskites, in addition to their stellar optoelectronic properties $^{3}$, arise from their facile and low-cost solution processability ${ }^{4}$. Spin coating is a well-established and widely utilized method for the formation of high-quality perovskite thin films. In recent years, improvements over conventionally spincoated thin film morphologies were achieved by exploiting treatment methods such as Lewis acid-base precursor adduct engineering ${ }^{5}$, complex intermediate driven crystallization ${ }^{6}$, additive usage $\mathrm{e}^{7}$, intramolecular exchange ${ }^{8}$, and antisolvent ${ }^{9}$ driven film formation. Post deposition film treatments such as thermal ${ }^{10}$ and solvent-vapor annealing ${ }^{11}$ have also been explored to eliminate undesirable ${ }^{12}$ structural constraints and are also known to lead to advantageous morphological effects such as the emergence of hierarchical microstructures within thin films ${ }^{13}$. Significant efforts toward controlling perovskite thin-film qualities have been undertaken because of the correlation ${ }^{14}$ between device performance metrics and thin-film structural characteristics. Moreover, a growing appreciation of the degree to which the structural quality of thin films is determined during the initial kinetic processing of colloidal precursors has instigated a close study of the evolving processing-structure-property relationships within thin films.

Synchrotron-based in situ X-ray ${ }^{15}$ methods have aided in elucidating the structural transformations occurring during the processing of hybrid perovskites, owing to high scattering length densities ${ }^{15}$ of heavy lead and halide-containing molecules. While $\mathrm{X}$-ray based characterizations are well suited for kinetic structure analysis, they provide limited insights on the functional response of the materials themselves. By utilizing the characteristically strong photoresponse of the materials, in situ optical methods ${ }^{16,17}$ provide insight into the evolving optoelectronic properties of crystallizing perovskites. The interdependence of structure sizes and their optical response especially helps in understanding the evolving nature ${ }^{18}$ of short-lived intermediates and the transformation kinetics ${ }^{19,20}$ between material phases. Important considerations that have emerged from the above studies include the identification of the complex sol-gel ${ }^{21}$ structures involved in the transformation, intermediate solventcomplex phases 22 involved during the assembly crystallization process ${ }^{23}$, the kinetics of their transformation to other phases $^{24-27}$, the impact of varying the time of antisolvent dispensing 28 , the importance of thermal annealing processes $^{27,29,30}$, and the sensitivity toward environmental conditions on the structure of processed thin films. Correlation of material structure and properties is usually established postfabrication and ex situ, where measured material properties can be strongly affected by differences between in situ and ex situ environments and by the impact of other synergistic functional materials involved. Structural and physical attributes attained during material formation highly influence subsequent material properties and provide information for further ex situ investigations. The sensitivity of out-of-equilibrium physicochemical structures to the multi-dimensional space of available experimental conditions ${ }^{31}$ makes the conclusive correlation of experimental observations to their phenomenological origins complex and time-intensive, and sometimes only accessible for observation with the development of new instrumentation.

In this work, by combining the complementary and reinforcing nature of information divulged by synchrotron radiation-based $\mathrm{X}$-ray and optical metrologies within controllable in situ processing environments, we unite processing-structure-function relationships. Herein the optoelectronic response is measured by means of photoluminescence (PL) spectroscopy tracking the varying functional optical response of changing structural entities traced by means of grazing-incidence wide-angle X-ray scattering (GIWAXS), during material processing by spin coating, antisolvent drop, and subsequent annealing to unveil previously empirically inaccessible mechanistic insights of complex colloidal crystallization.

Spin-coating is a solution-based processing method, which produces non-equilibrium thin films. The crystallization of colloidal precursors of hybrid perovskites has an inherent multivariate nature and is known to lead to reproducibility issues of the film characteristics. Routes of crystal growth have been discussed ${ }^{32}$, depicting how starting from generic precursor molecules, materials crystallize to their bulk form through multiple reaction pathways. Complementary and multimodal metrological techniques help to elucidate complex transformation mechanisms responsible for reproducibility issues. For instance, it is possible for the precursor to follow physicochemical growth pathways such as spinodal decomposition ${ }^{33}$, which deviate from the reaction pathways involving traditionally nucleated species. Rather, for instance, chemical reactions may proceed through the formation of intermediates, which convert to their final structural form on further treatment. Conceptualizing an understanding of complex growth processes requires the fixing of processing parameters, which we have done in the present study. In this work, we actuate the advantages of real-time investigation of the evolving structure and optoelectronic properties by combining GIWAXS and PL spectroscopy while emulating the one-step

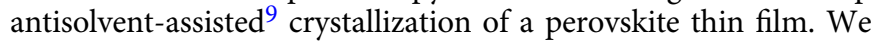
chose to investigate methylammonium lead iodide $\left(\mathrm{CH}_{3} \mathrm{NH}_{3} \mathrm{PbI}_{3}\right.$, MAPI), whose structural intermediates and transformation kinetics have been extensively investigated. This was done as a manner of demonstrating that much remains to be learned about out-of-equilibrium assembly processes of exemplary model systems. We report on the characteristics of the metastable structure formed by the first-order phase transition occurring during antisolvent-induced nucleation from the colloidal precursor sol. Further, there is a partial transformation of the metastable nuclei by concatenation of nanostructures leading to the formation of a solvent complex, with the solvent complex being stable against thermally induced degradation up to $100^{\circ} \mathrm{C}$. Around $100^{\circ} \mathrm{C}$, a second-order transformation process of the solvent complex to MAPI is initiated, by evaporation of the solvent from the thin film. Physicochemical reaction gradients are emergent and are attributed to differential rates of removal of strongly coordinated solvent molecules via advection of the evaporating solvent molecules from the film thickness. This mechanism of solvent removal results in a process of dissolution-recrystallization to lead to the final MAPI thin film. Further annealing leads to a ripening process of the crystalline film. Evolutionary data signatures, physical concepts, and characteristics within structure-function correlations learned from a model system of MAPI are transferable to other chemical compositions of hybrid perovskites. These insights are enabled through the development of a novel analytical cell ${ }^{34}$, which allows complete remote control over the spin coating process, antisolvent drop, PL excitation, and the annealing protocols. The processing and measurement environments are housed within an inert gas-purged cell to curb unfavorable degradation by atmospheric oxygen and moisture ${ }^{35}$.

\section{Results}

A simultaneous overview of the evolution of structural and optoelectronic phases recorded by GIWAXS and PL is presented in Fig. 1. Key structural phase transitions witnessed in diffraction are presented as individual 2D diffraction images in Fig. 2. 


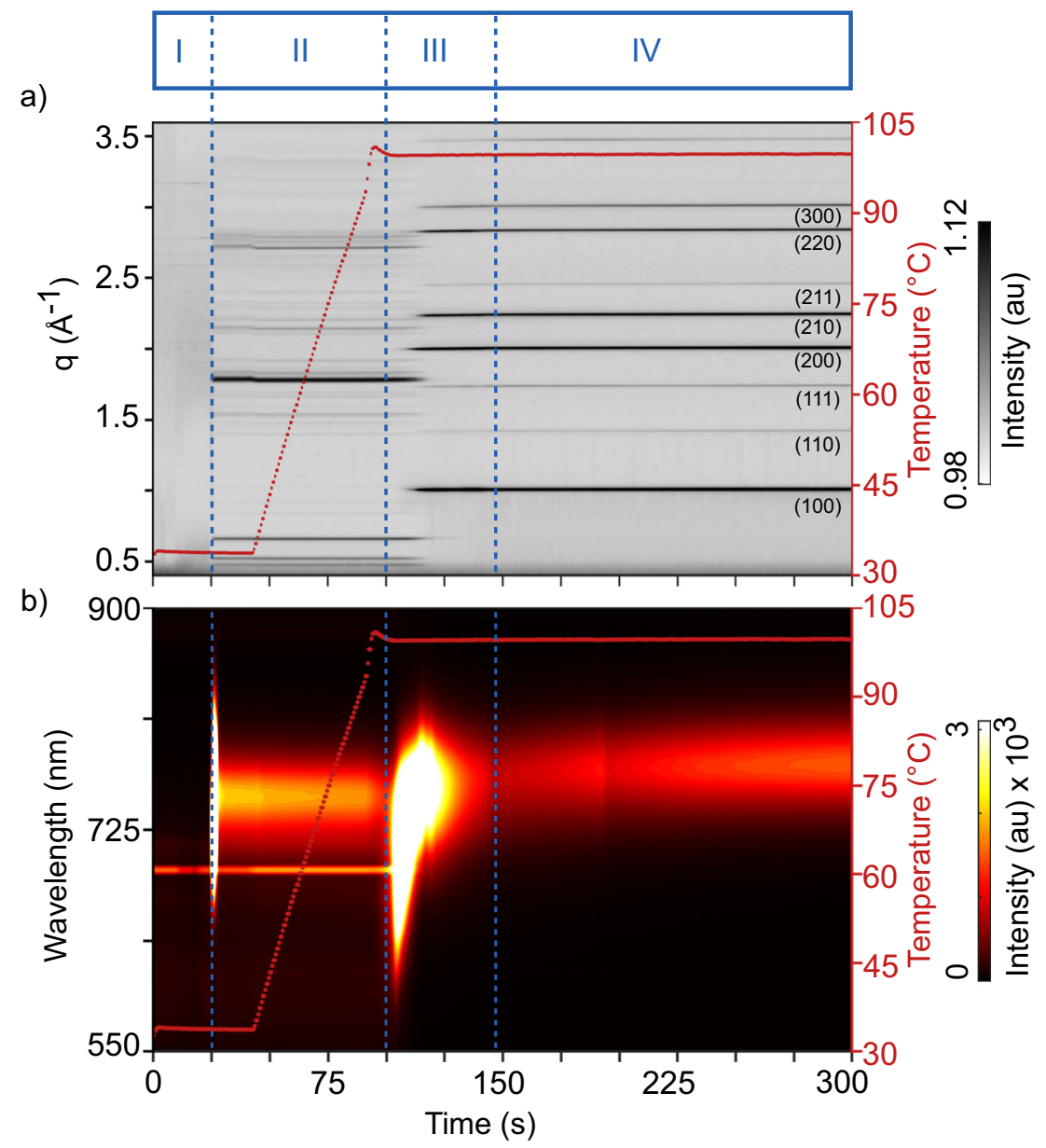

Fig. 1 Mapping with four phases seen in GIWAXS and PL data. Time evolution representing the four phases of the colloidal conversion process of $\mathrm{Pbl}_{2} . \mathrm{CH}_{3} \mathrm{NH}_{3}$ I.DMSO.DMF precursor to a final crystalline MAPI thin film as indicated by vertical dotted lines. a Radially integrated GIWAXS data as a function of q position and (b) PL data as a function of wavelength together with the substrate temperature (right $y$-axis). The narrow line emission at $690 \mathrm{~nm}$ is related to the diffuse reflection of the laser used in the position alignment system of the beamline. Phase I shows the spin coating followed by the antisolvent drop at $t=25 \mathrm{~s}$. In phase II, we observe diffraction from $\mathrm{Pb}_{3} \mathrm{I}_{8} .2\left(\mathrm{CH}_{3}\right)_{2} \mathrm{SO}_{2} 2 \mathrm{CH}_{3} \mathrm{NH}_{3}(\mathrm{MAPI}$.DMSO) solvent-complex phase, which is converted into MAPI crystals in phase III during annealing.

a)

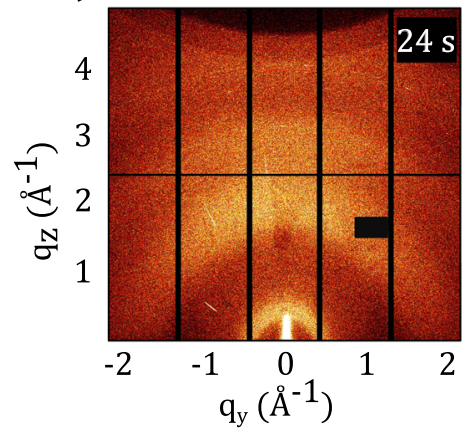

b)

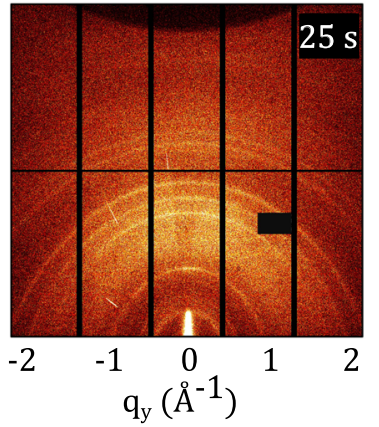

c)

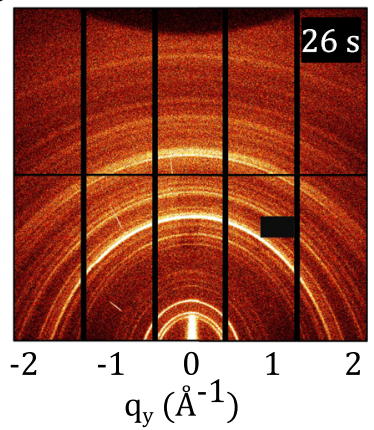

Fig. 2 Structural conversion seen in 2D GIWAXS data. 2D GIWAXS data as a function of the scattering vector components $q_{y}$ and $q_{z}$ during structural conversion of MAPI thin film (a) before, (b) during and (c) after the antisolvent drop at $t=25 \mathrm{~s}$. Diffraction from (a) colloidal mixture of solid precursors and solvent molecules, (b) instantly nucleated crystalline MAPI nanocrystals and nutrient colloidal phase in the slight background, and (c) crystalline orthorhombic MAPI.DMSO solvent-complex.

During the experiment, four equilibrium phases and three transient conversion processes are registered as explained below.

Phase I. In the first phase (phase I, $t=0-24 \mathrm{~s}$ ), the liquid precursor is spin-coated and reflected as diffuse halos centered around $0.5 \AA^{-1}, 1.8 \AA^{-1}$, and $3.0 \AA^{-1}$ (Fig. 2a) in the diffraction data. In line with other reports ${ }^{21,22,36}$, this signal is attributed to scattering from the mixture of the solvent and solute phases, composed of a colloidal dispersion of chemically and structurally diverse plumboiodide scattering species ${ }^{37,38}$. The low scattering contrast of the halos signifies the well-intermixed state of the 
solutes and solvents and the lack of any Bragg diffraction confirms the absence of long-range order within the precursor state. Phase I does not show any PL response (Fig. 1b).

Following $24 \mathrm{~s}$ of spin coating, an antisolvent stream is dynamically dispensed (the film is in spinning motion), resulting in rapid structural changes within the precursor phase. The diffuse halos from the precursor phase convert into low albeit distinct intensities with Bragg peaks located at $q=1.01,1.78$, $2.00,2.24,2.84$, and $3.01 \AA^{-1}$ (Fig. 2b). The amorphous background and low intensities of the Bragg peaks suggest an incomplete conversion of the colloidal precursor to the proceeding state. The film is found to be isostructural in terms of peak positions with cubic MAPI. The texture of the nucleating structure is attributed to the directionality of the antisolvent dispensing, which was set normal to the substrate plane. The diffraction signal from the nucleating phase quickly transforms to the proceeding state (phase II) (Fig. 2c) suggesting the metastable nature of the causal nucleating structure (Fig. 2b).

Phase II. Starting at $26 \mathrm{~s}$, phase II is initiated and the weak diffraction intensities from the metastable nucleated phase are converted into stronger diffraction intensities at $0.46 \AA^{-1}, 0.51 \AA^{-1}$, $0.65 \AA^{-1}, 1.75 \AA^{-1}$ together with several subsidiary peaks with lower intensities (Fig. 1a and Fig. 2c). The diffraction peaks correspond to the orthogonal crystalline solvent-complex, $\mathrm{Pb}_{3} \mathrm{I}_{8} .2\left(\mathrm{CH}_{3}\right)_{2} \mathrm{SO} .2 \mathrm{CH}_{3} \mathrm{NH}_{3}$ (MAPI.DMSO) ${ }^{39-41}$. In agreement with other reports ${ }^{42,43}$, no DMF-based solvent complexes are observed in spite of the precursor solvent being DMF-rich, due to the stronger Lewis acid-base complexing ability of $\mathrm{DMSO}^{5,44}$. No uncomplexed $\mathrm{PbI}_{2}$ is observed either. At the end of the spin coating process, at $44 \mathrm{~s}$, the annealing of the thin film is initiated with a linear temperature ramp of $1^{\circ} \mathrm{C} / \mathrm{s}$ to convert the solvent complex to the crystalline perovskite. During the rest of the annealing process, the peaks from the solvent-complex of phase II remain unchanged in terms of peak positions, intensities and widths, until a temperature of $100^{\circ} \mathrm{C}$ is reached and stabilized at $\sim 100 \mathrm{~s}$.

The antisolvent drop $(t=25 \mathrm{~s})$ triggers the immediate emergence of an intense and broad PL peak (Fig. 1b) centered at around $730 \mathrm{~nm}(1.70 \mathrm{eV})$. It is suggested that the PL emission arises from the instantly formed MAPI nanocrystals, (Fig. 2b) with a polydisperse size distribution of luminescing moieties responsible for the broad FWHM of the PL emission. The peak position, which in first approximation represents the bandgaps ${ }^{45,46}$, indicates quantum confinement of the charge carriers as typically observed in nanoparticles with a size range within ten nanometers ${ }^{47-50}$ (expected room temperature bandgap of bulk MAPI is $\sim 1.60 \mathrm{eV}^{51,52}$ ).

Within the next second, the peak position shifts towards $750 \mathrm{~nm}(1.65 \mathrm{eV})$ (Figure S1a) and remains constant afterward in phase II (Fig. 3d). The redshift is due to a growth in the size of nanocrystallites, leading to a reduction in the extent of quantum confinement ${ }^{50,53}$. The bulk bandgap is not reached, signifying an arrested growth mechanism after a few seconds, due to reaction and diffusion-limited constraints ${ }^{54,55}$ of the solvent-complex structures, which require thermal annealing to complete the solvent-evaporation and the transformation of the solventcomplex to crystalline perovskite. The FWHM of the PL peak at $750 \mathrm{~nm}$ shows significant narrowing from $130 \mathrm{meV}(t=26 \mathrm{~s})$ to $110 \mathrm{meV}(t=29 \mathrm{~s})$ (Figure S1a), corroborating the homogenization in size distribution. This occurs through an increase in the average sizes of structures formed by cluster coalescence of the nanoparticles, which have high correlated surface and interfacial energies. The high surface and interfacial energies are possibly strong driving forces for the cluster coalescence of the nucleated species due to their high surface-to-volume ratio. Coalescence results in size homogenization of the particles, which results in a narrowing of the PL spectra. After the polydisperse nucleation process, the MAPI nuclei above a critical radius are expected to remain stable against cluster coalescence. These MAPI crystals, which are not bound into the solvent complex phase are hypothesized to contribute to the remaining luminescence intensity in phase II (the solvent complex by itself likely does not luminesce). Thereafter, in phase II, the PL response remained unaffected in terms of peak shape, position, and intensity, suggesting remnants of stable perovskite crystals formed during the nucleation process.

Phase III. On reaching $100^{\circ} \mathrm{C}$, around $t=90 \mathrm{~s}$, the solvent complex undergoes further structural transitions caused by thermal disassociation and subsequent solvent evaporation, marking the initiation of phase III of the crystallization process. Around 103 s, Bragg peaks from a crystallizing perovskite phase start quickly gaining intensity, reaching the maximum at $120 \mathrm{~s}$ (Fig. 3a). The evolution of the perovskite phase (black curve) is compared against structural changes of the solvent-complex (green curve) by tracking the intensities of their Bragg peak $q_{100}=1.00 \AA^{-1}$ and $q_{150}=1.78 \AA^{-1}$ (Fig. 3a), respectively. The lattice spacings of the perovskite crystals increase while the peak widths narrow, for $t=104-120 \mathrm{~s}$ (Fig. 3b). Simultaneously, the solvent-complex Bragg peaks diminish in intensity. This relative intensity change between $104-120 \mathrm{~s}$ is attributed to the conversion of the solvent complex to the perovskite state at the film-air interface where the rate of evaporation of the solvent is expected to be the highest.

Beyond $120 \mathrm{~s}$, there is a second, slow decrease in the diffraction intensities of the solvent-complex (Fig. 3a) up to $144 \mathrm{~s}$, which is attributed to the removal of the solvent complex from the deeper parts of the thin film, which require longer annealing times for complete solvent removal. Such an observation confirms insights on the structural gradients of the structure within thin films ${ }^{56}$, where crystallization occurs at different rates within varying thicknesses of the film by solvent evaporation and interdiffusion ${ }^{57}$ and resultant colloidal assembly ${ }^{58}$. Moreover, the Bragg peaks of the solventcomplex lose intensity from the off-normal orientations faster, and the remaining intensities of the Bragg peaks from the solventcomplex display orientations dominantly normal to the film substrate (Figure S2). With continued annealing, the perovskite film crystal orientation becomes increasingly mosaic as seen from the increasing widths of the related Bragg peaks, and the homogenized distribution of the preferential orientation spread of the perovskite Bragg peaks (Figure S2b, c). During the gradual removal of the solvent complex, the intensity of the perovskite peak (Fig. 3a) shows distinct fluctuations, first diminishing ( $t=121-130 \mathrm{~s})$, then increasing $(t=131-136 \mathrm{~s})$, and then decreasing again $(t=137-144 \mathrm{~s})$. These fluctuations are attributed to dissolving and recrystallizing previously formed perovskite crystals as the solvent molecules from deeper interfaces are removed by solvent mass transfer and crystalline rearrangement through the already crystallized film thickness. The peak width of the perovskite crystals during this stage ( $t=121-144 \mathrm{~s}$ ) increases while the lattice parameters reflect a slight decrease (Fig. 3b), confirming the presence of processes that engender increased structural disorder within the thin film. Any discernible signs from the solventcomplex phase disappear as the film is fully converted into the dry crystalline phase (phase IV, $t=144 \mathrm{~s}$ ).

Upon reaching $100{ }^{\circ} \mathrm{C}$ in phase $\mathrm{III}$, a second bright PL response emerges between 650 and $740 \mathrm{~nm}(1.68-1.9 \mathrm{eV})$, with its center around $1.72 \mathrm{eV}$ and a FWHM of $190 \mathrm{meV}$ (Fig. 3d) observed at $t=104 \mathrm{~s}$ (Figure $\mathrm{S} 1 \mathrm{~b}$ ). This signature is attributed to the 

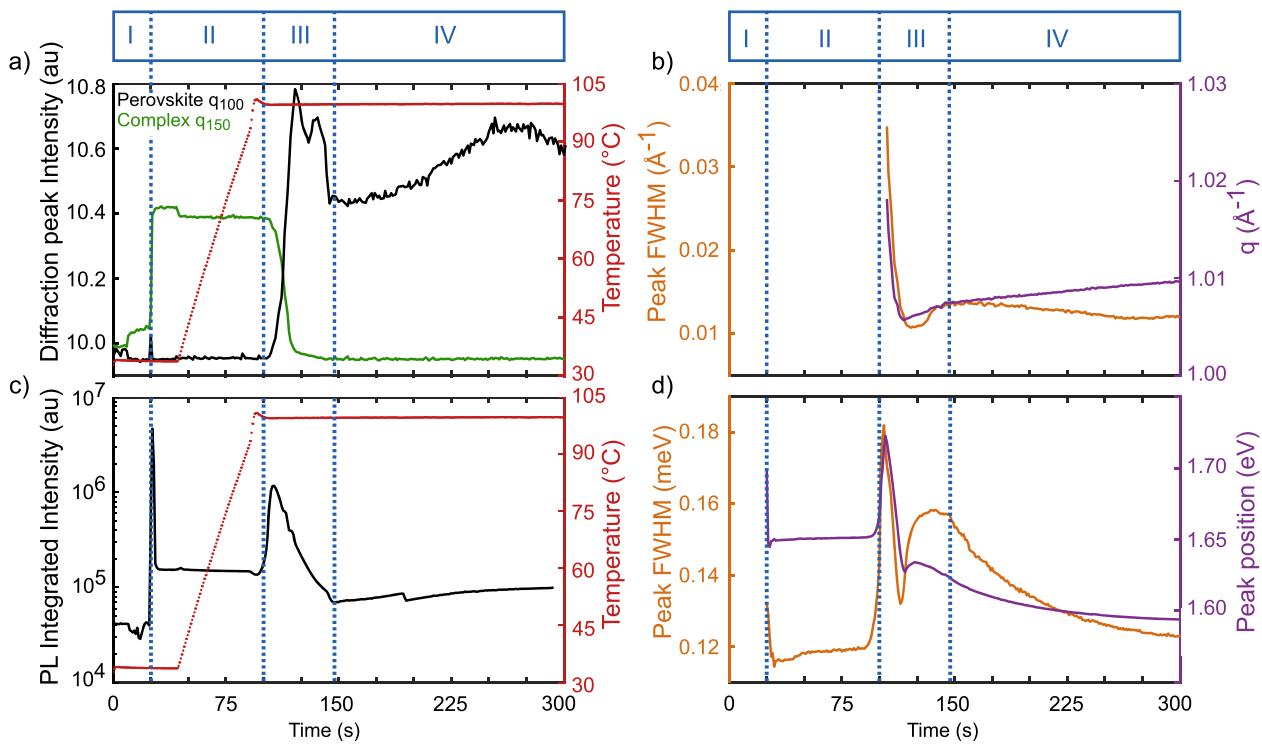

Fig. 3 Temporal evolution of characteristic parameters. a The temporal evolution of radially integrated GIWAXS data of MAPI (black) and MAPI.DMSO solvent-complex (green) as well as temperature (red) and (b) perovskite lattice parameters peak full width at half maximum (FWHM, orange) and q position (purple) during the crystallization process of perovskite solvent complex at $100{ }^{\circ} \mathrm{C}$. c Integrated PL emission intensity (black) during nucleation, annealing, and dissolution-recrystallization processes of thin film as well as temperature (red) and (d) Evolution of PL peak parameters peak FWHM (orange) and position (purple) during crystallization experiment. The four phases are indicated with vertical dotted lines.

co-existence of disparate nanocrystallite sizes with high radiative efficiency. The non-Gaussian peak shape comes from a superposition of luminescence signals with disparate intensity contributions ${ }^{47,59}$. Akin to the processes occurring during the moments proceeding antisolvent dispensing $(t>25 \mathrm{~s})$, the PL signal intensity decreases and redshifts, indicating growth of the nanocrystallites. The underlying growth kinetics are slower, while the redshift of the peak maximum is more significant, reaching $1.72 \mathrm{eV}$ at $t=103 \mathrm{~s}$. In parallel, the peak width decreases from $180 \mathrm{meV}(t=103 \mathrm{~s})$ to $130 \mathrm{meV}(t=114 \mathrm{~s})$ (Fig. $3 \mathrm{~d})$. After the peak width attains a local minimum $(t=114 \mathrm{~s})$, a subsequent rebroadening to $158 \mathrm{meV}(t=135 \mathrm{~s})$ is observed, correlated to a small peak position shift towards higher energies. These trends reflect the dissolution and creation of small clusters with higher bandgap, as the solvent from the deeper parts are removed, confirming the trends in the diffraction data.

Phase IV. The final phase IV ( $t>144 \mathrm{~s})$ represents exclusive diffraction signals from cubic MAPI at $q=1.00,1.42,1.74,2.00$, $2.24,2.46,2.84,3.01$, and $3.48 \AA^{-1}$. In this phase, the intensity of the perovskite peak increases (Fig. 3a) up to $260 \mathrm{~s}$, while the peak width narrows (Fig. 3b), suggesting enhanced crystallinity and reduced lattice strain on longer annealing. The Bragg peak intensity distribution suggests a similar slight preferred orientation (Fig. S2c) of the crystals of the thin film normal to the plane of the substrate, as is observed within the transient structure (Fig. 2b) when the antisolvent is dispensed. Beyond $260 \mathrm{~s}$, the peak intensity of the perovskite decreases slightly (Fig. 3a) as the peak width saw a slight increase, which might be indicative of the onset of beam damage. No $\mathrm{PbI}_{2}$ is isolated although the perovskite peak broadening signifies increased disorder in crystals.

In phase IV, the gradual increase in the overall PL intensities (Fig. 3c) is accompanied by an FWHM narrowing and shift in peak position towards $780 \mathrm{~nm}(1.60 \mathrm{eV})$, representative of luminescence from a stabilized bulk MAPI (Fig. 3d). PL data however did not indicate beam damage. Long-term annealing and cooling of a sample reflect the presence of a $\mathrm{PbI}_{2}$ phase $\left(q \sim 0.9 \AA^{-1}\right)$ as well as of a tetragonal MAPI phase $\left(q \sim 1.4 \AA^{-1}\right)$ (Fig. S3).

\section{Discussion}

While there are several reports on phase transformations and identification $20,21,28,60$, detailed insights into the phenomena occurring at critical synthesis and phase transition stages are desirable. Importantly, the kinetic processes occurring during nucleation and dissolution-recrystallization have not been revealed in detail so far, and are the focus of the present study (Fig. 4).

Within the context of crystallization of colloidal systems from solutions, the crystallization processes are known to proceed by fluctuating solvodynamics resulting in initial "low-amplitude", longwavelength density fluctuations ${ }^{61,62}$ through a large volume, followed by the actual crystallization event (Fig. S4c). These fluctuations may be reflected in our diffraction data right before the emergence of the weak Bragg reflections from the supersaturated phase when the scattering signal shows an intermediate transition from colloidal halos (Fig. S4a) at $q=0.5,1.8$, and $3.0 \AA^{-1}$ to a broad background signal (Fig. S4b) at the moment the antisolvent is dropped to initiate the crystallization process. Chemically, by virtue of the high miscibility of chlorobenzene with DMSO and DMF 9 and its poor solubility with the perovskite solid precursors, a phase separation process occurs as excess solvent molecules are displaced from the sample by the antisolvent stream, ensuing a marked increase in the concentration of the solute species within the system creating the conditions for a phase transition process to transpire. This phase transformation process, which marks the phase boundary between the fluid colloidal precursor to the gel intermediate state, can proceed by one of two routes, namely LaMer nucleation $^{63-65}$ or kinetically arrested spinodal decomposition ${ }^{66}$. Both processes signify pathways of segregation and evolution of a new phase ${ }^{67}$ from a melt, where nucleation-driven phase transitions have associated activation energy for the creation of metastable nuclei, which coalesce to form the solvent-complex intermediate. A kinetically arrested spinodal decomposition process leads to the 


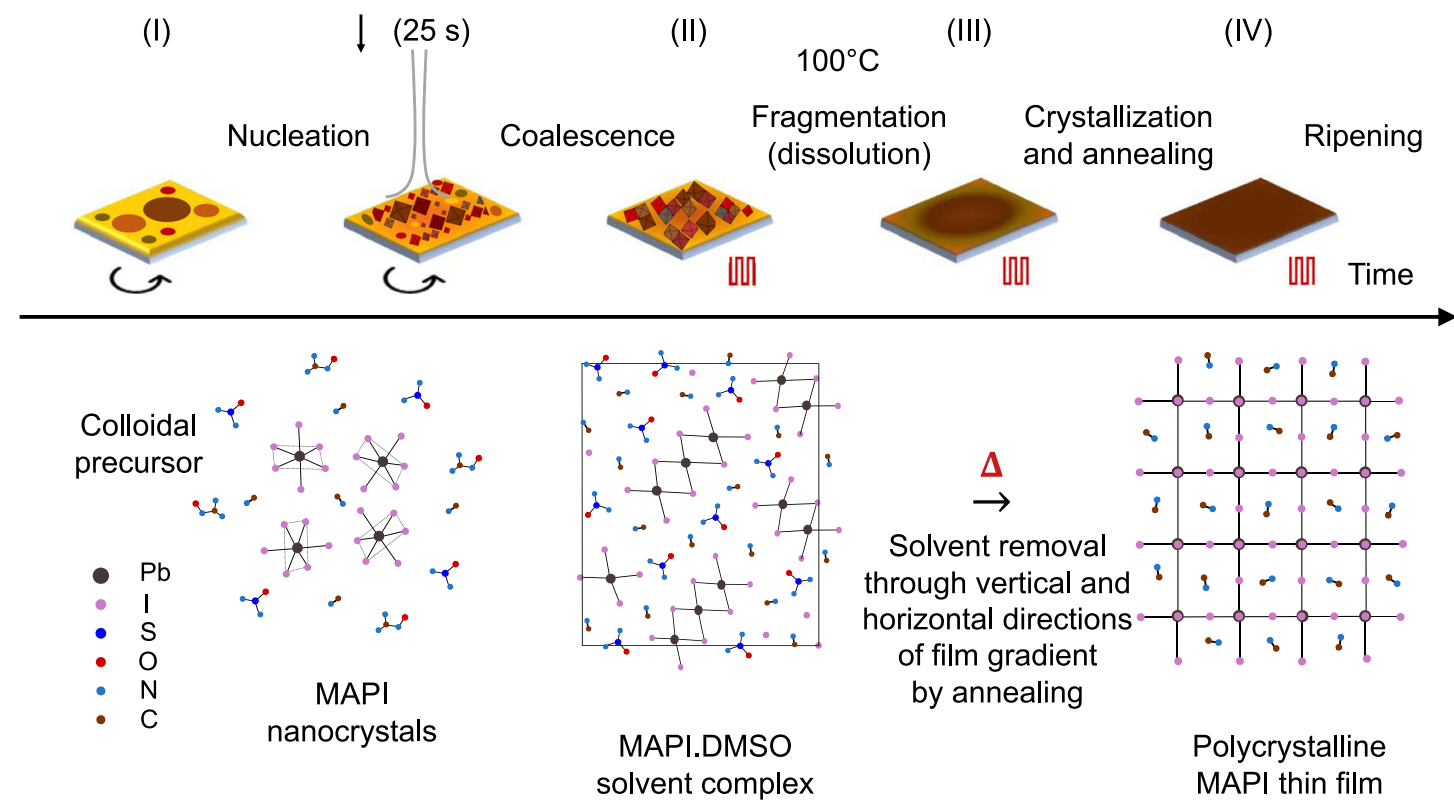

Fig. 4 Four phases of film formation during spin coating. Phase I: The thin film consists of colloidal precursors of photoinactive solid precursors ( $\mathrm{Pbl} \mathrm{I}_{2}$ and $\left.\mathrm{CH}_{3} \mathrm{NH}_{3} \mathrm{I}\right)$ and liquid solvents $\left(\mathrm{CH}_{3}\right)_{2} \mathrm{~N}-\mathrm{HCO}$ and $\left(\mathrm{CH}_{3}\right)_{2} \mathrm{SO}$. Nucleation of MAPI nanocrystals and conversion to phase II where the nanoparticles trimerize into thermodynamically stable orthorhombic solvent-complex of $\mathrm{Pb}_{3} \mathrm{I}_{8} \cdot 2\left(\mathrm{CH}_{3}\right)_{2} \mathrm{SO}_{2} 2 \mathrm{CH}_{3} \mathrm{NH}_{3}$ with remainders of stable perovskite phase formed during nucleation. Phase III: Thermal decomposition of solvent-complex leading to the removal of plumboiodide coordinated solvent molecules at $100{ }^{\circ} \mathrm{C}$ from the thin film and the eventual dissolution-recrystallization of $\mathrm{CH}_{3} \mathrm{NH}_{3} \mathrm{Pbl}_{3}$ from the film thickness. Phase IV: Perovskite crystallization complete and thermal ripening process of the thin film.

spontaneous formation of the said intermediate without the creation of metastable nuclei due to fluctuations in the energetics of the precursor. The impact of varying the antisolvent drop time during spin-coating strongly affects the material conversion pathways and resulting morphologies. Previous studies have extensively studied the impact of drop times and formulated and explained the concept of antisolvent drop time windows in separate studies ${ }^{19,28,68}$. In the absence of an antisolvent, materials crystallizing out of a fluid precursor nucleate and crystallize in a broad time window, whereas the application of an orthogonal solvent is a physical route to temporally regulate supersaturation and initiate growth during material crystallization/formation. Accordingly, there are distinct differences in precursor chemistry and methods of physicochemical conversion between a one-step ${ }^{9}$ and two-step ${ }^{20,69}$ method which are typically discussed in the literature. The one-step method combines the organic $\left(\mathrm{CH}_{3} \mathrm{NH}_{3} \mathrm{I}\right)$ and inorganic precursors $\left(\mathrm{PbI}_{2}\right)$ in a solvent system (4:1 v:v DMF:DMSO) to afford a single colloidal precursor. This precursor is spin-coated into a thin film, followed by an application of an antisolvent and subsequent thermal annealing to result in a $\mathrm{CH}_{3} \mathrm{NH}_{3} \mathrm{PbI}_{3}$ film. The two-step method proceeds via the separated treatment of the inorganic $\left(\mathrm{PbI}_{2}\right)$ precursor solution-processed into a thin film with suitable organic solvents (GBL, DMF, DMSO), immersed into an organic precursor $\left(\mathrm{CH}_{3} \mathrm{NH}_{3} \mathrm{I}\right.$ /isopropanol) to convert to $\mathrm{CH}_{3} \mathrm{NH}_{3} \mathrm{PbI}_{3}$ via thermal annealing driven interdiffusion method. The removal of the bulk solvent molecules is expected to lead to a drastic reduction in the excluded free volume available for the solute molecules and cause a pinned gel-like structure ${ }^{21,66}$ with decreased diffusion abilities. The stochastic nature ${ }^{61,70}$ of nucleation of the perovskite precursor colloid $^{37,71}$, where a broad distribution of nuclei sizes are expected at supersaturation, is reflected in the evolution of the PL FWHM. Whereas antisolvent-driven nucleation is expected to lead to a homogenous nucleation event, experiments suggest that the actual nature of nucleation depends on the time window employed for antisolvent dispensing ${ }^{19}$. In our experiments, for the employed experimental conditions, an event resulting in a broad distribution of nanoparticles is marked, as witnessed by the broad photoluminescence signal. Such a signal implies a broad distribution in the density of states of luminescing species, which corroborate a distribution in the nuclei sizes formed during nucleation. We note that this is the first observation of the pre-nucleation density fluctuations, (Fig. S4b) and nucleation (Fig. 2b) within crystallizing perovskite systems by combined diffraction and photoluminescence data. The nuclei are notably isostructural with the perovskite (MAPI) phase but owing to the large destabilizing surface and interfacial energies associated, the diffraction data of the nucleated phase is only briefly observed (Fig. 2b) before converting to the solvent-complex phase (Fig. 2c). The nucleating species likely have high surface charge, and cluster-aggregation of individual monomers $\left(\mathrm{PbI}_{6}{ }^{4-}\right)$ to the trimerized orthogonal solvent complex $\left(\mathrm{Pb}_{3} \mathrm{I}_{8}{ }^{2-}\right)$ proceeds by means of increased entropy ${ }^{72}$ (and reduced free energy) on the release of solvating molecules during the postnucleation, early-growth stages. The DMSO molecules coordinate the trimerized aggregates, while the organic methylammonium $\left(\mathrm{CH}_{3} \mathrm{NH}_{3}{ }^{+}\right)$ions are known to characteristically direct the selfassembly ${ }^{73,74}$ of the structures resulting in the denser solventcomplex. The PL intensity is found to be influenced by two major factors: the nucleation density (total amount of perovskite material responding to the photoexcitation) and the extent of radiative recombination (depending on crystal quality and defect density ${ }^{26,53}$ ). The data supports the reduction in the polydispersity soon after the instance of nucleation by the growth in average particle size, as reflected within the narrowed shape of the PL peak and its redshift from $730-750 \mathrm{~nm}$. The PL intensity during antisolvent-induced nucleation increases $(t=25-27 \mathrm{~s})$ as shown in Fig. $3 c$, in agreement with previous reports ${ }^{53}$ and is ascribed to an increasing number of nanocrystallites ${ }^{50,53}$. Subsequently, the intensity is quenched $(30 \times)$ due to cluster coalescence; also reflected within the PL redshift. The coalesced clusters have reduced emission ${ }^{75}$ as compared to the nucleated nanoparticles because with size increase, structures have a higher probability for non-radiative recombinations at defect sites and grain boundaries. A combination 
of increased crystallite size and thermal quenching leads to an increase in the extent of non-radiative recombination and a resultant reduction in the luminescence intensity from the remaining stable MAPI crystals formed during nucleation 26,50.

The thermodynamic stability of the DMSO-complexated phase requires thermal annealing to drive the removal of the DMSO molecules. The reduced free solvent content within the film is associated with the limited diffusion ability of the media, until the energy barrier is overcome by heating the sample. Throughout the annealing process, the nature of the diffraction and photoluminescence signals remain largely unchanged, until $100^{\circ} \mathrm{C}$ is reached and the disintegration process of the solvent complex is initiated, marking a subsequent second-order phase transition. During thermal disassociation of the MAPI-DMSO solvent complex, the diffraction intensities from the solvent complex diminish as the diffraction intensity of the perovskite phase gains in intensity (Fig. 3a). While the diffraction data reveals the two coexistent phases as they evolve, marking the second order of the phase transition, the PL data yields mechanistic insights underlying the transition. The disassociation of the solvent complex leads to the removal of DMSO by evaporation, leaving behind fragmented building blocks of the crystallizing MAPI phase. This process is deemed responsible for the increased polydispersity of the molecular species, reflected in the broadened and markedly blue-shifted PL signal during phase III. Owing to the increased surface energies of the disassociated particles, the fragments thermally diffuse to form longer chains to reduce the total free energy of the system. This process of the concatenation of plumboiodide fragments and their assembly within crystalline cubic perovskite lattice are reflected by shifts in the PL signal. The PL signal after its previous broadening and blue shift, undergoes rapid narrowing and redshifts as solvent molecules are rapidly removed from the thin film leaving MAPI crystals behind. It has been found that owing to differential solvent evaporation rates from the film-air interface and the deeper entrenched solvent moieties, the film develops a vertical gradient of solvent distribution resulting in a crystallizing front leading from the filmair interface into the film-substrate interface, creating a crust of crystallized perovskite at the surface ${ }^{76}$.

The processes occurring during annealing and drying can be distinguished into distinct drying stages, with considerations of heat and mass transfer phenomena ${ }^{77}$. After the initiation of the annealing step in phase II, the temperature of the substrate increases linearly over time and by a heat-transfer process, the temperature of the thin film increases. In contrast, during phase III, the temperature is kept constant and the thermal disintegration of the solvent complex allows significant evaporation of the solvent to the air interface. The solvent removal falls within the "fast" regime ${ }^{78}$ and is strongly affected by the coupled heat and mass transfer between the drying film interface and air. The process of solvent removal proceeds by diffusion and evaporation and therefore includes advective mass transfer through the bulk. The removal of the solvent molecules underneath the film-air interface is responsible for the secondary processes beyond $120 \mathrm{~s}$, where both the PL and the diffraction peaks show fluctuations in intensity due to subsequent redissolution and crystallization within different depths and interfaces. Phase IV of the process of crystallization is marked by the full conversion of the solvent complex into the MAPI structure, with all structural and optoelectronic signatures of the complex disappearing, signifying the completion of the crystallization process.

In conclusion, the combination of in situ photoluminescence and grazing-incidence wide-angle $\mathrm{X}$-ray scattering is used to follow in real-time the colloidal processing of perovskite thin films during spin coating. Advanced multimodal experimental observation of the structure and optoelectronic properties of the luminescent, metastable nucleated $\mathrm{CH}_{3} \mathrm{NH}_{3} \mathrm{PbI}_{3}$ nanoparticles during processing is presented. These building blocks are tracked in real-time as they are transformed into the orthogonal solventcomplex $\mathrm{Pb}_{3} \mathrm{I}_{8} .2\left(\mathrm{CH}_{3}\right)_{2} \mathrm{SO} .2 \mathrm{CH}_{3} \mathrm{NH}_{3}$. During annealing, the solvent complex disintegrates, and a solvent gradient is established through the thin film leading to a crystallization-redissolutionrecrystallization and rearrangement process throughout the film thickness. The final phase of $\mathrm{CH}_{3} \mathrm{NH}_{3} \mathrm{PbI}_{3}$ is stabilized in the cubic symmetry and exhibits the expected structural and optoelectronic characteristics.

\section{Methods}

Multimodal experiment. The experiment was carried out at the 12.3.2 microdiffraction beamline at the Advanced Light Source in a custom-made analytical chamber, allowing for processing of the thin film and simultaneous multimodal measurements. The $\mathrm{TiO}_{2}$ covered plasma cleaned glass substrate was placed onto the integrated spin coating puck-heater and held in place by a heat transfer paste. A liquid precursor of $1 \mathrm{M} \mathrm{PbI}_{2}$ and $\mathrm{CH}_{3} \mathrm{NH}_{3} \mathrm{I}$ in a solvent mixture of 4:1 DMF:DMSO was pipetted and deposited onto the surface of the substrate and the chamber was sealed off from the external environment by being held under a nitrogen flow. The experiment was conducted by spin coating the precursor at two spin coating speeds, a first $10 \mathrm{~s}$ of slow rotation at $100 \mathrm{rpm}$ to ensure the uniform spread of the precursor onto the substrate and a second $30 \mathrm{~s}$ of fast rotation at $3000 \mathrm{rpm}$ in order to fabricate a thin film. $15 \mathrm{~s}$ into the second spin coating step $(t=25 \mathrm{~s})$, a stream of chlorobenzene was dispensed through a pre-programmed syringe pump. At the end of the spin coating protocol, a heating protocol was remotely initiated, in two stages. In the first stage ( $t=45-90 \mathrm{~s})$, the temperature was increased linearly at the rate of $1{ }^{\circ} \mathrm{C} / \mathrm{s}$ until it reached and stabilized at $100^{\circ} \mathrm{C}$. Thereafter, the temperature was maintained at $100^{\circ} \mathrm{C}$ until the end of the experiment $(t=90-300 \mathrm{~s})$. The incident angle of the incoming X-ray beam was $1^{\circ}$ with a beam energy of $10 \mathrm{keV}$. The sample detector distance (SDD) was $\sim 155 \mathrm{~mm}$ and the detector was positioned at an angle of $39^{\circ}$ from the sample plane.

In situ measurements. GIWAXS data were recorded every second on a 2D Pilatus $1 \mathrm{M}$ detector (Dectris Ltd.). Photoluminescence excitation was achieved through a $532 \mathrm{~nm}$ Thorlabs diode-pumped solid-state laser with a power density of $40 \mathrm{~mW} /$ $\mathrm{cm}^{2}$. The resultant photoluminescence signal was collected by a lens and focused on an optical fiber guiding it to a grating OceanOptics QE Pro spectrometer for detection. The temperature of the heating puck was recorded by a pre-calibrated Raytek MI3 pyrometer, which regulated the annealing temperature and protocol through a pre-programmed PID loop.

\section{Data availability}

The data are available from the corresponding authors upon reasonable request.

\section{Code availability}

The code used for data management and analysis are available from the corresponding author upon reasonable request.

Received: 30 November 2020; Accepted: 8 June 2021; Published online: 24 September 2021

\section{References}

1. Kim, H., Han, J. S., Choi, J., Kim, S. Y. \& Jang, H. W. Halide perovskites for applications beyond photovoltaics. Small Methods 2, 1700310 (2018).

2. Song, Z. et al. A technoeconomic analysis of perovskite solar module manufacturing with low-cost materials and techniques. Energy Environ. Sci. 10, 1297-1305 (2017).

3. Stranks, S. D., Hoye, R. L. Z., Di, D., Friend, R. H. \& Deschler, F. The physics of light emission in halide perovskite devices. Adv. Mater. 31, e1803336 (2019).

4. Dunlap-Shohl, W. A., Zhou, Y., Padture, N. P. \& Mitzi, D. B. Synthetic approaches for halide perovskite thin films. Chem. Rev. 119, 3193-3295 (2019).

5. Lee, J.-W., Kim, H.-S. \& Park, N.-G. Lewis acid-base adduct approach for high efficiency perovskite solar cells. Acc. Chem. Res. 49, 311-319 (2016).

6. Zhou, X. et al. Crystallization manipulation and morphology evolution for highly efficient perovskite solar cell fabrication via hydration water induced intermediate phase formation under heat assisted spin-coating. J. Mater. Chem. A 6, 3012-3021 (2018). 
7. Liang, P.-W. et al. Additive enhanced crystallization of solution-processed perovskite for highly efficient planar-heterojunction solar cells. Adv. Mater. 26, 3748-3754 (2014).

8. Yang, W. S. et al. SOLAR CELLS. High-performance photovoltaic perovskite layers fabricated through intramolecular exchange. Science 348, 1234-1237 (2015).

9. Jeon, N. J. et al. Solvent engineering for high-performance inorganic-organic hybrid perovskite solar cells. Nat. Mater. 13, 897-903 (2014).

10. Eperon, G. E., Burlakov, V. M., Docampo, P., Goriely, A. \& Snaith, H. J. Morphological control for high performance, solution-processed planar heterojunction perovskite solar cells. Adv. Funct. Mater. 24, 151-157 (2014).

11. Shao, Y., Yuan, Y. \& Huang, J. Correlation of energy disorder and open-circuit voltage in hybrid perovskite solar cells. Nat. Energy 1, 1-6 (2016).

12. Jones, T. W. et al. Lattice strain causes non-radiative losses in halide perovskites. Energy Environ. Sci. 12, 596-606 (2019).

13. Pratap, S., Schlipf, J., Bießmann, L. \& Muller-Buschbaum, P. Hierarchical structures from nanocrystalline colloidal precursors within hybrid perovskite thin films: implications for photovoltaics. ACS Appl. Nano Mater. 3, 11701-11708 (2020).

14. Leblebici, S. Y. et al. Facet-dependent photovoltaic efficiency variations in single grains of hybrid halide perovskite. Nat. Energy 1, 16093 (2016).

15. Schlipf, J. \& Müller-Buschbaum, P. Structure of organometal halide perovskite films as determined with grazing-incidence X-ray scattering methods. $A d v$. Energy Mater. 7, 1700131 (2017)

16. Babbe, F. \& Sutter-Fella, C. M. Optical absorption-based in situ characterization of halide perovskites. Adv. Energy Mater. 10, 1903587 (2020).

17. Buchhorn, M., Wedler, S. \& Panzer, F. Setup to study the in situ evolution of both photoluminescence and absorption during the processing of organic or hybrid semiconductors. J. Phys. Chem. A 122, 9115-9122 (2018).

18. van Franeker, J. J. et al. Monitoring thermal annealing of perovskite solar cells with in situ photoluminescence. Adv. Energy Mater. 7, 1601822 (2017).

19. Song, T.-B. et al. Dynamics of antisolvent processed hybrid metal halide perovskites studied by in situ photoluminescence and its influence on optoelectronic properties. ACS Appl. Energy Mater. 3, 2386-2393 (2020).

20. Chauhan, M. et al. Investigating two-step MAPbI 3 thin film formation during spin coating by simultaneous in situ absorption and photoluminescence spectroscopy. J. Mater. Chem. A 8, 5086-5094 (2020).

21. Wang, K. et al. Kinetic stabilization of the sol-gel state in perovskites enables facile processing of high-efficiency solar cells. Adv. Mater. 31, e1808357 (2019).

22. Hu, Q. et al. In situ dynamic observations of perovskite crystallisation and microstructure evolution intermediated from $\left[\mathrm{PbI}_{6}\right]^{4-}$ cage nanoparticles. Nat. Commun. 8, 15688 (2017).

23. Boles, M. A., Engel, M. \& Talapin, D. V. Self-assembly of colloidal nanocrystals: from intricate structures to functional materials. Chem. Rev. 116, 11220-11289 (2016).

24. McMeekin, D. P. et al. Crystallization Kinetics and Morphology Control of Formamidinium-Cesium Mixed-Cation Lead Mixed-Halide Perovskite via Tunability of the Colloidal Precursor Solution. Adv. Mater. https:/doi.org/ 10.1002/adma.201607039 (2017).

25. Moore, D. T. et al. Crystallization kinetics of organic-inorganic trihalide perovskites and the role of the lead anion in crystal growth. J. Am. Chem. Soc. 137, 2350-2358 (2015).

26. Suchan, K., Just, J., Becker, P., Unger, E. L. \& Unold, T. Optical in situ monitoring during the synthesis of halide perovskite solar cells reveals formation kinetics and evolution of optoelectronic properties. J. Mater. Chem. A 8, 10439-10449 (2020).

27. Barrows, A. T. et al. Monitoring the formation of a $\mathrm{CH} 3 \mathrm{NH} 3 \mathrm{PbI} 3-\mathrm{x} \mathrm{Cl} x$ perovskite during thermal annealing using X-ray scattering. Adv. Funct. Mater. 26, 4934-4942 (2016)

28. Bruening, K. \& Tassone, C. J. Antisolvent processing of lead halide perovskite thin films studied by in situ X-ray diffraction. J. Mater. Chem. A 6, 18865-18870 (2018).

29. Nenon, D. P. et al. Structural and chemical evolution of methylammonium lead halide perovskites during thermal processing from solution. Energy Environ. Sci. 9, 2072-2082 (2016).

30. Yang, Y. et al. Annealing Induced Re-crystallization in CH3NH3PbI3-xClx for High Performance Perovskite Solar Cells. Sci. Rep. 7, 46724 (2017).

31. Gu, E. et al. Robot-Based High-Throughput Screening of Antisolvents for Lead Halide Perovskites. Joule; https://doi.org/10.1016/j.joule.2020.06.013 (2020).

32. Yoreo, J. Jde et al. Crystallization by particle attachment in synthetic, biogenic, and geologic environments. Science 349, aaa6760 (2015).

33. Binder, K. \& Fratzl, P. Spinodal Decomposition. In Phase transformations in materials, edited by G. Kostorz (Wiley-VCH, Weinheim, New York, Chichester, 2010), pp. 409-480.

34. Pratap, S. et al. Probing the in situ dynamics of structure-property evolution in hybrid perovskite thin films spincoated from complex fluids by a custom- designed beamline-compatible multimodal measurement chamber. Acta Cryst. A 75, a155-a156 (2019)

35. Schlipf, J. et al. In situ monitoring the uptake of moisture into hybrid perovskite thin films. J. Phys. Chem. Lett. 9, 2015-2021 (2018).

36. Qin, M. et al. Manipulating the mixed-perovskite crystallization pathway unveiled by in situ GIWAXS. Adv. Mater. 31, e1901284 (2019).

37. Pratap, S., Keller, E. \& Müller-Buschbaum, P. Emergence of lead halide perovskite colloidal dispersions through aggregation and fragmentation: insights from the nanoscale to the mesoscale. Nanoscale 11, 3495-3499 (2019).

38. Yan, K. et al. Hybrid halide perovskite solar cell precursors: colloidal chemistry and coordination engineering behind device processing for high efficiency. J. Am. Chem. Soc. 137, 4460-4468 (2015)

39. Cao, J. et al. Identifying the molecular structures of intermediates for optimizing the fabrication of high-quality perovskite films. J. Am. Chem. Soc. 138, 9919-9926, https://doi.org/10.1021/jacs.6b04924 (2016).

40. Guo, Y. et al. Chemical pathways connecting lead(ii) iodide and perovskite via polymeric plumbate(ii) fiber. J. Am. Chem. Soc. 137, 15907-15914 (2015).

41. Rong, Y. et al. Solvent engineering towards controlled grain growth in perovskite planar heterojunction solar cells. Nanoscale 7, 10595-10599 (2015).

42. Dang, H. X. et al. Multi-cation synergy suppresses phase segregation in mixedhalide perovskites. Joule 3, 1746-1764 (2019).

43. Hamill, J. C., Schwartz, J. \& Loo, Y.-L. Influence of solvent coordination on hybrid organic-inorganic perovskite formation. ACS Energy Lett. 3, 92-97 (2018).

44. Zhang, H., Nazeeruddin, M. K. \& Choy, W. C. H. Perovskite photovoltaics: the significant role of ligands in film formation, passivation, and stability. Adv. Mater. 31, e1805702 (2019).

45. Gfroerer, T. H. Photoluminescence in Analysis of Surfaces and Interfaces (American Cancer Society, 2006).

46. Unold, T. \& Gütay, L. Photoluminescence Analysis of Thin-Film Solar Cells. In Advanced Characterization Techniques For Thin Film Solar Cells, edited by D. Abou-Ras, T. Kirchartz \& J. Rau (Wiley-VCH, Weinheim, 2016 WileyVCH Verlag Weinheim), pp. 275-297.

47. Parrott, E. S. et al. Growth modes and quantum confinement in ultrathin vapour-deposited MAPbI3 films. Nanoscale 11, 14276-14284 (2019).

48. Nie, W. et al. Solar cells. High-efficiency solution-processed perovskite solar cells with millimeter-scale grains. Science 347, 522-525 (2015).

49. D'Innocenzo, V., Srimath Kandada, A. R., Bastiani, Mde, Gandini, M. \& Petrozza, A. Tuning the light emission properties by band gap engineering in hybrid lead halide perovskite. J. Am. Chem. Soc. 136, 17730-17733 (2014).

50. Wagner, L. et al. Distinguishing crystallization stages and their influence on quantum efficiency during perovskite solar cell formation in real-time. Sci. Rep. 7, 14899 (2017).

51. Shirayama, M. et al. Optical transitions in hybrid perovskite solar cells: ellipsometry, density functional theory, and quantum efficiency analyses for CH3NH3PbI3. Phys. Rev. Appl. 5, 14012 (2016).

52. Fujiwara, H., Kato, M., Tamakoshi, M., Miyadera, T. \& Chikamatsu, M. Optical characteristics and operational principles of hybrid perovskite solar cells. Phys. Status Solidi A 215, 1700730 (2018).

53. Song, T.-B. et al. Revealing the dynamics of hybrid metal halide perovskite formation via multimodal in situ probes. Adv. Funct. Mater. 30, 1908337 (2020).

54. Meakin, P. Models for colloidal aggregation. Annu. Rev. Phys. Chem. 39, 237-267 (1988).

55. Thanh, N. T. K., Maclean, N. \& Mahiddine, S. Mechanisms of nucleation and growth of nanoparticles in solution. Chem. Rev. 114, 7610-7630 (2014).

56. Schaefer, C., Michels, J. J. \& van der Schoot, P. Dynamic surface enrichment in drying thin-film binary polymer solutions. Macromolecules 50, 5914-5919 (2017).

57. Tsige, M. \& Grest, G. S. Solvent evaporation and interdiffusion in polymer films. J. Phys. Condens. Matter 17, S4119-S4132 (2005).

58. Howard, M. P. et al. Evaporation-induced assembly of colloidal crystals. J. Chem. Phys. 149, 94901 (2018).

59. Li, J., Dobrovolsky, A., Merdasa, A., Unger, E. L. \& Scheblykin, I. G. Luminescent intermediates and humidity-dependent room-temperature conversion of the MAPbI3 perovskite precursor. ACS Omega 3, 14494-14502 (2018).

60. Mundt, L. E. \& Schelhas, L. T. Structural evolution during perovskite crystal formation and degradation: in situ and operando X-ray diffraction studies. Adv. Energy Mater. 10, 1903074 (2020).

61. Lutsko, J. F. A dynamical theory of nucleation for colloids and macromolecules. J. Chem. Phys. 136, 34509 (2012).

62. Lutsko, J. F. \& Lam, J. Long-wavelength density fluctuations as nucleation precursors. Phys. Rev. E 101, 52122 (2020).

63. LaMer, V. K. \& Dinegar, R. H. Theory, production and mechanism of formation of monodispersed hydrosols. J. Am. Chem. Soc. 72, 4847-4854 (1950).

64. $\mathrm{Hu}, \mathrm{H}$. et al. Nucleation and crystal growth control for scalable solutionprocessed organic-inorganic hybrid perovskite solar cells. J. Mater. Chem. A 8 , 1578-1603 (2020). 
65. Liu, C., Cheng, Y.-B. \& Ge, Z. Understanding of perovskite crystal growth and film formation in scalable deposition processes. Chem. Soc. Rev. 49, 1653-1687 (2020).

66. Gibaud, T. \& Schurtenberger, P. A closer look at arrested spinodal decomposition in protein solutions. J. Phys. Condens. Matter 21, 322201 (2009).

67. Schmelzer, J. W. P., Abyzov, A. S. \& Möller, J. Nucleation versus spinodal decomposition in phase formation processes in multicomponent solutions. $J$. Chem. Phys. 121, 6900-6917 (2004).

68. Zhao, P. et al. Antisolvent with an ultrawide processing window for the onestep fabrication of efficient and large-area perovskite solar cells. Adv. Mater. 30, e1802763 (2018).

69. Xiao, Z. et al. Efficient, high yield perovskite photovoltaic devices grown by interdiffusion of solution-processed precursor stacking layers. Energy Environ. Sci. 7, 2619-2623 (2014).

70. Lutsko, J. F. How crystals form: A theory of nucleation pathways. Sci. Adv. 5, eaav7399 (2019).

71. Dutta, N. S., Noel, N. K. \& Arnold, C. B. Crystalline Nature of Colloids in Methylammonium Lead Halide Perovskite Precursor Inks Revealed by CryoElectron Microscopy. J. Phys. Chem. Lett. 5980-5986; https://doi.org/10.1021/ acs.jpclett.0c01975 (2020).

72. Habraken, W. J. E. M. et al. Ion-association complexes unite classical and nonclassical theories for the biomimetic nucleation of calcium phosphate. Nat. Commun. 4, 1507 (2013)

73. Shevchenko, E. V., Talapin, D. V., Murray, C. B. \& O’Brien, S. Structural characterization of self-assembled multifunctional binary nanoparticle superlattices. J. Am. Chem. Soc. 128, 3620-3637 (2006).

74. Lobo, RaulF., Zones, StaceyI. \& Davis, MarkE. Structure-direction in zeolite synthesis. J. Incl. Phenom. Macrocycl. Chem. 21, 47-78 (1995).

75. van Dijken, A., Makkinje, J. \& Meijerink, A. The influence of particle size on the luminescence quantum efficiency of nanocrystalline $\mathrm{ZnO}$ particles. J. Lumin. 92, 323-328 (2001).

76. Chen, A. Z. et al. Understanding the formation of vertical orientation in twodimensional metal halide perovskite thin films. Chem. Mater. 31, 1336-1343 (2019).

77. Velaga, S. P., Nikjoo, D. \& Vuddanda, P. R. Experimental studies and modeling of the drying kinetics of multicomponent polymer films. AAPS PharmSciTech 19, 425-435 (2018).

78. Guerrier, B., Bouchard, C., Allain, C. \& Bénard, C. Drying kinetics of polymer films. AIChE J. 44, 791-798 (1998)

\section{Acknowledgements}

This work was supported by funding from TUM.solar in the context of the Bavarian Collaborative Research Project Solar Technologies Go Hybrid (SolTech) and by the Deutsche Forschungsgemeinschaft (DFG, German Research Foundation) under Germany's Excellence Strategy - EXC 2089/1 - 390776260 (e-conversion). S.P. acknowledges support from the TUM International Graduate School of Science and Engineering (IGSSE) via the GreenTech Initiative Interface Science for Photovoltaics (ISPV) of the EuroTech Universities, the Bavaria California Technology Center (BaCaTeC), and the Centre for Nanoscience (CeNS). Special thanks are extended to the Nanosystems Initiative Munich (NIM) and the generous ALS Doctoral Fellowship in Residence for funding and administrative support that made this work possible. H. Goudey and the ALS workshop are acknowledged for their support with the development of the instrument. Dr. A. Sharma and L. P. Kreuzer helped with their critical insights. This research used resources of the Advanced Light Source, a U.S. DOE Office of Science User Facility under contract no. DE-AC02-05CH11231. This manuscript was prepared with support from the Laboratory Directed Research and Development (LDRD) program of Lawrence Berkeley National Laboratory under U.S. Department of Energy Contract DEAC02-05CH11231(T.-B.S. and C.M.S.-F.). This material is based upon work performed by the Joint Center for Artificial Photosynthesis, a DOE Energy Innovation Hub, supported through the Office of Science of the U.S. Department of Energy under Award DESC0004993 (F.B.). C.M.S.-F. acknowledges the Molecular Foundry supported by the Office of Science, Office of Basic Energy Sciences, of the U.S. Department of Energy under Contract No. DE-AC02-05CH11231.

\section{Author contributions}

C.M.S.-F., C.V.S., N.T., S.P. and J.L.S. conceived, designed, built, and deployed the instrumentation. S.P., N.S.B., Z.H., Z.Y., T.L., T.-B.S., J.L.S., C.V.S., N.T. and C.M.S.-F. conducted the experiment. C.V.S. and N.T. provided beamtime supervision and support. S.P., Z.Y., N.S.B. and F.B. developed methods for data analysis and representation. S.P., F.B., C.M.S.-F. and P.M.B. wrote the manuscript. C.M.S.-F. and P.M.B. supervised the project. All authors discussed and approved the final paper.

\section{Funding}

Open Access funding enabled and organized by Projekt DEAL.

\section{Competing interests}

The authors declare no competing interests.

\section{Additional information}

Supplementary information The online version contains supplementary material available at https://doi.org/10.1038/s41467-021-25898-5.

Correspondence and requests for materials should be addressed to Carolin M. Sutter-Fella or Peter Müller-Buschbaum.

Peer review information Nature Communications thanks Thomas Riedl and other, anonymous, reviewers for their contributions to the peer review of this work. Peer review reports are available.

Reprints and permission information is available at http://www.nature.com/reprints

Publisher's note Springer Nature remains neutral with regard to jurisdictional claims in published maps and institutional affiliations.

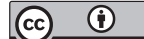

Open Access This article is licensed under a Creative Commons Attribution 4.0 International License, which permits use, sharing, adaptation, distribution and reproduction in any medium or format, as long as you give appropriate credit to the original author(s) and the source, provide a link to the Creative Commons license, and indicate if changes were made. The images or other third party material in this article are included in the article's Creative Commons license, unless indicated otherwise in a credit line to the material. If material is not included in the article's Creative Commons license and your intended use is not permitted by statutory regulation or exceeds the permitted use, you will need to obtain permission directly from the copyright holder. To view a copy of this license, visit http://creativecommons.org/ licenses/by/4.0/.

(C) The Author(s) 2021 